\title{
Sobre los golpes de calor en "hot cars" como atentado contra animales no humanos, a propósito de dos casos penales en Chile
}

\author{
José Ignacio Binfa Álvarez \\ Abogado Universidad Mayor \\ Diplomado en Criminología Pontificia Universidad Católica \\ Director Fundación Abogados por los Animales \\ Orcid https://orcid.org/0000-0002-2524-6379
}

Cita recomendada. BINFA ÁLVAREZ, J.I., Sobre los golpes de calor en "hot cars" como atentado contra animales no humanos, a propósito de dos casos penales en Chile, dA. Derecho Animal (Forum of Animal Law Studies) 12/1 (2021). - DOI https://doi.org/10.5565/rev/da.559

Agradecimientos. Quiero agradecer a la médica veterinaria María Francisca Arce Valdés, colaboradora de Fundación Abogados por los Animales, con quien pude profundizar los aspectos veterinarios de la temática abordada en este trabajo.

\section{Resumen}

El presente trabajo ofrece un análisis jurídico de la conducta de dejar animales en el interior de un vehículo en condiciones climáticas que produzcan un golpe de calor, situación que ha sido denominada como "hot cars" en el mundo anglosajón y que puede constituir delito de maltrato de animal. Se presentan dos casos que han abordado esta problemática en Chile y se analiza la regulación de esto en EE. UU, para concluir con una propuesta de lege ferenda que haga frente a esta situación.

Palabras claves: derecho animal; derecho penal; maltrato animal; animales de compañía; hot cars.

Abstract - On heatstroke in "hot cars" as assault against non-human animals, regarding two criminal cases in Chile

This paper offers a legal analysis of the behaviour of leaving animals inside a vehicle in weather conditions that cause heatstroke; a situation that has been called "hot cars" in the Anglo-Saxon world, and can constitute an animal abuse crime. Two cases in Chile that have addressed this problem are presented, and its regulation in the US is analysed, to conclude with a proposal de lege ferenda that deals with this issue.

Keywords: animal law; criminal law; animal abuse; companion animals; hot cars. 


\section{Sumario}

1. Introducción

2. Casos

3. Comentario de los casos

4. Una mirada al exterior: regulación de "hot cars" en EE.UU

5. Conclusiones

\section{Introducción}

Cada verano, las altas temperaturas y el comportamiento descuidado de cuidadores de animales de compañía, generan situaciones en que estos últimos son expuestos de forma prolongada a altas temperaturas en espacios cerrados, terminando gravemente afectados por golpes de calor y en algunos casos muertos. Estos hechos, ocurren principalmente en vehículos motorizados estacionados, lo cual genera alarma en la sociedad chilena, pues cada verano somos testigos de casos de esta naturaleza ${ }^{1}$, motivando a expertos, medios de comunicación y a organizaciones de protección animal, a entregar información sobre cómo prevenir y afrontar estos eventos ${ }^{2}$.

Desde el punto de vista veterinario ${ }^{4}$, el golpe de calor es una condición que genera trastornos fisiológicos en animales. En el caso puntual de individuos caninos y felinos, cuando la temperatura corporal de éstos asciende a $41^{\circ} \mathrm{C}$, se genera el síndrome de shock térmico, asociado a una hipertermia que comienza a generar diferentes trastornos orgánicos tales como: deshidratación, fallas renales o sistémicas generales, vómitos, anomalías cardiovasculares, entre otras. A nivel etológico, generan ansiedad, malestar y sufrimiento a los animales no humanos que lo padecen.

En el ámbito jurídico, este tipo de conductas pueden llegar a constituir delito de maltrato de animal, figura delictiva que en Chile está tipificada en los artículos 291 bis y ter del Código Penal ${ }^{5}$ y esta última norma

\footnotetext{
${ }^{1}$ Además de los casos que se expondrán a continuación, es posible en contra en la prensa chilena numerosos hechos de esta naturaleza. A modo de ejemplo:

SoyQuillota (2020): Justo a tiempo: rescatan a cachorro encerrado en vehículo a pleno sol en La Ligua. En línea: https://www.soychile.cl/Quillota/Sociedad/2020/12/31/687868/Justo-a-tiempo-rescatan-a-cachorro-encerrado-en-vehiculo-a-plenosol-en-La-Ligua.aspx [último acceso el 16 de enero de 2021].

El Ovallino (2020): Detenido por maltrato animal al dejar a su mascota dentro del auto. En línea:

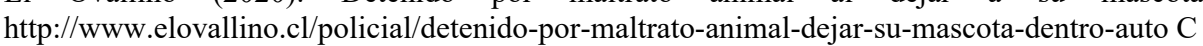

Cooperativa (2020): Rescatan a cachorro que estaba encerrado en un auto expuesto al intenso calor. En línea: $\mathrm{https://cooperativa.cl/noticias/pais/region-de-valparaiso/rescatan-a-cachorro-que-estaba-encerrado-en-un-auto-expuesto-al-}$ intenso/2020-12-28/115610.html [último acceso el 16 de enero de 2021].

Mestizos Magazine (2019): Mujer deja a su perro encerrado en un auto en Conchalí. En línea: https://mestizos.cl/noticias/mujer-dejaa-su-perro-encerrado-en-un-auto-en-conchali/ [último acceso el 16 de enero de 2021].

Mestizos Magazine (2019): Perro permaneció encerrado por más de una hora en un auto. En línea: https://mestizos.cl/noticias/perropermanecio-encerrado-por-mas-de-una-hora-en-un-auto/ [último acceso el 16 de enero de 2021].

BioBio Chile (2019): "Oye, es mi auto. No lo rompan por favor": dejó encerrado a su perro con $30^{\circ} \mathrm{C}$ y fue detenida. En línea: https://www.biobiochile.cl/noticias/nacional/region-de-la-araucania/2019/02/01/oye-es-mi-auto-no-lo-rompan-por-favor-dejoencerrado-a-su-perro-con-30o-de-calor-y-fue-detenida.shtml [último acceso el 16 de enero de 2021].

${ }^{2} \mathrm{La}$ Tercera (2020): Ola de calor: cómo cuidar a tus mascotas frente a las altas temperaturas. En línea: https://www.latercera.com/practico/noticia/ola-de-calor-como-cuidar-a-tus-mascotas-frente-a-las-altastemperaturas/XSYJS244FNGYBCOWNONZHSL32E/ [último acceso el 16 de enero de 2021].

${ }^{3}$ Colegio Médico Veterinario $(\mathrm{s} / \mathrm{f})$ : Cómo Evitar la Insolación en Perros. En línea: https://www.colegioveterinario.cl/noticias/ver.php?id=194 [último acceso el 16 de enero de 2021].

${ }^{4}$ ARCE VALDÉS, M. F., comunicación personal, 17 de diciembre de 2020.

${ }^{5}$ Artículo 291 bis del Código Penal: "El que cometiere actos de maltrato o crueldad con animales será castigado con la pena de presidio menor en sus grados mínimo a medio y multa de dos a treinta unidades tributarias mensuales, o sólo con esta última. Si como resultado de una acción u omisión se causare al animal daño, la pena será presidio menor en sus grados mínimo a medio y multa de diez a treinta unidades tributarias mensuales, además de la accesoria de inhabilidad absoluta perpetua para la tenencia de cualquier tipo de animales. Si como resultado de las referidas acción u omisión se causaren lesiones que menoscaben gravemente la integridad física o provocaren la muerte del animal se impondrá la pena de presidio menor en su grado medio y multa de veinte a treinta unidades tributarias mensuales, además de la accesoria de inhabilidad absoluta perpetua para la tenencia de animales." Artículo 291 ter del Código Penal: "Para los efectos del artículo anterior se entenderá por acto de maltrato o crueldad con animales toda acción u omisión, ocasional o 
define a los actos de maltrato o crueldad como "toda acción u omisión, ocasional o reiterada, que injustificadamente causare daño, dolor o sufrimiento al animal". La pena de este delito va de entre 61 días a 3 años de presidio, además de multa y accesoria de inhabilidad absoluta perpetua para la tenencia de animales dependiendo del resultado. Este es un delito cuyo bien jurídico -a la luz de las reformas introducidas en 2017 por la ley $\mathrm{N}^{\circ} 21.020$ - es la integridad y vida del animal no humano ${ }^{6}$, en cuanto este último titular de dichos bienes $^{7}$, por lo que conductas como dejar a un animal no humano expuesto a las altas temperaturas en espacios cerrados, claramente ponen en riesgo e incluso pueden lesionar dichos bienes jurídicos, sin perjuicio que se trata de un delito de lesión y, consecuentemente, la simple puesta en peligro no sería punible. Es un delito que, de acuerdo con su definición, puede ser realizado por acción u omisión ${ }^{8}$, siendo además un delito que exige la producción de un resultado típico ${ }^{9}$, el cual, en la figura base, es daño, dolor o sufrimiento, mientras que en la figura agravada del inciso tercero del artículo 291 bis del Código Penal corresponde a lesiones que menoscaben gravemente la integridad física o provocaren la muerte del animal. Aterrizando esto al problema del presente trabajo, el resultado de la prolongada exposición al calor es el golpe de calor o shock térmico e indudablemente nos encontramos ante una situación que genera daño, dolor y sufrimiento a un animal no humano e incluso puede provocarle la muerte.

Por otro lado, en cuanto al elemento subjetivo de este delito, la norma no contempla exigencias especiales, haciendo admisible tanto su comisión con dolo directo o eventual. Respecto de la posibilidad de una realización culposa o imprudente de este tipo penal, se debe destacar que, en la legislación chilena, rige un sistema numerus clausus de delitos culposos, estableciéndose en el artículo $10 \mathrm{~N}^{\circ} 13$ del Código Penal ${ }^{10}$, por regla general, que estos estarán exentos de responsabilidad, salvo en aquellos casos que expresamente la ley los castigue. De este modo, el maltrato de animal culposo no se castiga en Chile al no existir una norma expresa que lo tipifique.

En este contexto normativo, en Chile son escasos los pronunciamientos judiciales sobre este tipo de casos, sin contar con sentencias condenatorias a la fecha, ni menos con normativa especial para abordar esta problemática. Como este problema no es exclusivo de este país, en derecho comparado podemos encontrar respuestas normativas para este tipo de casos. Así, un ejemplo de esto es el caso de los EE. UU, país en el cual esta problemática se denomina "hot cars" organización Animal Legal Defense Fund (en adelante: ALDF), existirían al menos tres tipos de legislaciones estatales en los EE. UU según la forma en que abordan el problema de los "hot cars"12.

A continuación, se expondrán y analizarán dos casos ocurridos en Chile relacionados a causaciones de muerte a animales de compañía producto del encierro de ellos en vehículos motorizados. Luego, se revisará brevemente la experiencia de los EE. UU respecto del tema, para, finalmente, entregar en las conclusiones algunas ideas que puedan servir para un debate de lege ferenda respecto de la problemática de los "hot cars" en nuestro país.

\section{Casos}

\subsection{Caso 1: RUC No 1900082897-4 de Rengo}

Este caso ocurrió en enero de 2019 en la comuna de Rengo, Región del Libertador Bernardo O'Higgins, y tuvo cobertura en medios de la localidad ${ }^{13}$ en atención a que afectó a dos canes, uno de ellos falleció y hubo detención en flagrancia. El procedimiento inicia por denuncia de un testigo del lugar, que alerta a la policía,

reiterada, que injustificadamente causare daño, dolor o sufrimiento al animal."

${ }^{6}$ BINFA ÁLVAREZ, J., Delito de maltrato animal en el Anteproyecto de Nuevo Código Penal de Chile de 2018 , dA. Derecho Animal (Forum of Animal Law Studies) 11/3 (2020) 143.

${ }^{7}$ MAÑALICH, J. P., Animalidad y Subjetividad. Los animales (no humanos) como sujetos-de-derecho, en Revista de Derecho (Valdivia). Volumen 31, $\mathrm{N}^{\circ} 2$ (2018) 324.

${ }^{8}$ Atrás quedó la discusión respecto de si el verbo rector "cometer" se opone a formas de comisión por omisión de este delito y que, con el tiempo, fue reconociendo su punibilidad gracias a leyes como la $\mathrm{N}^{\circ} 20.380$ que establecieron expresamente posiciones de garante. Sobre esto, vid., GUZMÁN DÁLBORA, J., El delito de maltrato de animales, en Estudios Penales (Santiago, 2009$) 279-280$. ${ }^{9}$ Previo a las reformas introducidas por la ley $\mathrm{N}^{\circ} 21.020$, se discutía si este era un delito de mera actividad o de resultado. Ya anterior a estas reformas, GUZMÁN DALBORA explicaba que el maltrato animal es un delito de resultado. Vid., GUZMÁN DÁLBORA, J., op. cit., pp. 278-279.

${ }^{10}$ Artículo 10 del Código Penal: "Están exentos de responsabilidad criminal:

13. 'El que cometiere un cuasidelito, salvo en los casos expresamente penados por la ley."

${ }^{11}$ Animal Legal Defense Fund (2019): Acts against leaving dogs locked in hot cars. En línea: https://aldf.org/project/dogs-in-hot-cars/ [último acceso el 16 de enero de 2021].

${ }^{12}$ Animal Legal Defense Fund (s/f): An Avoidable Tragedy: Dogs in Hot Cars. En línea: https://aldf.org/project/an-avoidable-tragedydogs-in-hot-cars/ [último acceso el 16 de enero de 2021].

${ }^{13}$ El Tipógrafo (2019): Rengo: Denuncian grave caso de maltrato animal contra dos perritas que dejaron encerradas en auto. En línea: https://eltipografo.cl/2019/01/rengo-denuncian-grave-caso-de-maltrato-animal-contra-dos-perritas-que-dejaron-encerradas-en-auto [último acceso el 16 de enero de 2021]. 
quienes concurren al lugar y proceden al rescate de los canes forcejeando una de las puertas, aprehendiendo a los imputados. De esta forma, el día 22 de enero de 2019 comienza un procedimiento ordinario por delito de maltrato de animal contra los imputados de iniciales L. E. C. C. y G. E. A. C., en causa RIT 347-2019 RUC 1900082897-4 ante el Juzgado de Garantía de Rengo, quienes en la audiencia de control de detención quedaron en libertad, sujetos a las medidas cautelares de firma mensual y arraigo nacional.

Durante el procedimiento, no hubo querellantes y el día 9 de abril de 2019 el Ministerio Público presenta acusación, remitiéndose la competencia al Tribunal de Juicio Oral en lo Penal de Rancagua, para conocer el juicio oral en procedimiento ordinario bajo el RIT 341-2019.

\subsubsection{Hechos de la acusación fiscal}

"El día 21 de enero del año 2019 en horas de la mañana L. E. C. C. y G. E. A. C. llegaron en el vehículo marca Kia de propiedad de don L. E. C. C. hasta la calle Manuel Solís de la ciudad de Rengo, dejando estacionado ese vehículo en el lugar, en cuyo interior se encontraban dos cachorros caninos de 3 meses de vida aproximadamente el vehículo se encontraba totalmente cerrado solamente con una de las ventanas mínimamente abierta, no obstante existir una alta temperatura ambiental. Siendo las 12:40 aproximadamente una persona que pasaba por el lugar, doña I. L. C. advirtió el llanto de los animales y los golpes de uno de estos en el maletero del vehículo provocando que personal policial llegara hasta el lugar y buscara la forma de lograr rescatar a los animales, toda vez que uno de ellos ya había perdido el conocimiento encontrándose al interior del mismo, después de algunos minutos de trabajo se logró abrir la puerta del costado derecho del vehículo y se rescato a los dos perros, uno de ellos fue trasladado de urgencia a la clínica veterinaria Las Nieves, la perra mestiza de 3 meses, hembra, que presentaba pérdida de conciencia, no estaba atenta al medio, presentaba mucosas cianóticas, frecuencia cardíaca elevada a una temperatura corporal de 42 grados Celsius, convulsionando en dos oportunidades sin recobrar conciencia, vomitando también en al menos una oportunidad, falleciendo en horas de la noche producto de una hipertermia a causa del golpe de calor prolongado, mientras que el otro animal quedó al cuidado de una tercera persona, este animal se encuentra estable y en proceso de hidratación." (sic)

\subsubsection{Resolución del caso}

El día 6 de septiembre de 2019, se celebra el juicio oral, en el cual se rindieron las pruebas y los magistrados emitieron su veredicto. A juicio del Tribunal de Juicio Oral en lo Penal, los hechos de la acusación del Ministerio Público resultaron acreditados con la prueba rendida, especialmente con los testimonios de la testigo denunciante, los funcionarios policiales y el médico veterinario que atendió al can que falleció, dándose por establecidos los hechos y la participación de los acusados.

No obstante, la mayoría del tribunal sostuvo que la prueba rendida fue insuficiente para probar el elemento subjetivo exigido por este delito, es decir, dolo eventual. Para llegar a esta conclusión, señalaron en su veredicto que la imputación a título de dolo exigiría que los acusados, a lo menos, se representaran que la conducta realizada (esto es, dejar a los canes en el interior de un vehículo en un día que se registró una alta temperatura) podría ocasionar el resultado típico y haber aceptado este resultado, cuestión que en este caso no pudo ser probada más allá de toda duda razonable. Para el voto mayoritario, que los acusados hayan efectuado acciones para resguardar a los animales, como dejar las ventanas semi abiertas y que uno de los canes no haya fallecido, sería coherente con que los acusados no habrían obrado con dolo eventual como exige el tipo penal de maltrato de animal. De este modo, el resultado de este caso fue absolutorio.

En cuanto a la sentencia, el considerando séptimo se encargaría de analizar el elemento subjetivo del tipo penal, reafirmando lo señalado en el veredicto y añadiendo que las acciones para resguardar a los animales que habrían realizado los acusados dejarían en evidencia que, si bien se representaron el resultado, este no habría sido aceptado, obrando de esta forma con culpa con representación. Por otro lado, también influiría en esta decisión el hecho que el otro can haya resultado ileso. De este modo, el tribunal razona que "si bien pudieron representarse que la acción de dejarlos en dicho lugar podía ocasionarles algún daño, confiaron en que el mismo sería evitado si dejaban abierta una de sus ventanas" y de este modo estaría ausente el elemento subjetivo que exigiría este tipo penal aún en su figura base del inciso primero del artículo 291 bis, fundando así la absolución de los acusados.

En el fallo, también se pronunciaría un voto en contra, que estaría por la condena por delito de maltrato de animal, pero no en la hipótesis originalmente planteada por el Ministerio Público, es decir, inciso tercero del artículo 291 bis por el resultado de muerte, sino que por la figura base del inciso primero del mismo artículo. Para el magistrado que emite este voto, en lo que respecta al elemento subjetivo ciertamente se puede descartar la concurrencia de dolo directo, pero subsistiría dolo eventual pues claramente los sujetos se 
representan la posibilidad de un resultado lesivo para los canes (daño, dolor o sufrimiento), y actuaron de todas formas aceptando o siendo indiferente a las consecuencias. En palabras del magistrado, los sujetos habrían decidido actuar pese al hipotético resultado, pensando "nos vamos a almorzar dejando los cachorros expuestos pase lo que les pase". En opinión de este juez, de conformidad a la dinámica de los hechos, en ningún caso los acusados se podrían haber representado la muerte de alguno de los perros, pues dejaron las ventanas semi abiertas, y lo reprochable sería que se representaron la desesperación y sufrimiento que padecieron los perros al quedarse encerrados, siéndoles esto indiferente. Para finalizar, el magistrado advierte: "Una pregunta ayuda en muchos casos a resolver un dilema, como en este caso ¿hubieran dejado a un niño encerrado en esas circunstancias? Lo más probable es que no, precisamente por el riesgo que había, pero como eran animales sí lo hicieron, contraviniendo lo que el legislador, recogiendo un clamor social, quiso tutelar." No obstante este voto minoritario, Ministerio Público no interpuso recursos contra el fallo, quedando firme y ejecutoriado.

\subsection{Caso 2: RUC No 1910003650-1 de Viña del Mar}

Este caso obtuvo más atención mediática ${ }^{14}$ y fue denominado como "caso Castaña" por las organizaciones que intervinieron en él. Este hecho ocurrió el 19 de enero de 2019 en la comuna de Viña del Mar, Región de Valparaíso, y tuvo una particularidad: un tercero, cuidador de vehículos del sector donde estaba estacionado el automóvil en el cual se encontraba Castaña, una perra adulta tipo Golden retriever, procede a romper con una piedra uno de los vidrios del vehículo para rescatar al can, pero producto del golpe de calor ya se encontraba sin vida. La persona que trató de rescatar a Castaña alertó a las policías, quienes llegaron al lugar y lo tomaron detenido por el delito de daños por dicho acto ${ }^{15}$.

El procedimiento corresponde al RIT 1063-2019, iniciado por querella presentada por Municipalidad de Viña del Mar el día 22 de enero de 2019, a la cual se sumaría con posterioridad querella de la Fundación Derecho y Defensa Animal, detallando la dinámica de los hechos. Con posterioridad, el Ministerio Público presentaría una solicitud de audiencia de formalización, la cual se realizaría el 21 de noviembre de 2019. En la formalización, se le comunicaron los cargos al imputado de iniciales C. A. M. V., no se decretaron medidas cautelares y se fijaría audiencia de suspensión condicional del procedimiento, la cual se realizó el día 2 de diciembre de 2019.

\subsubsection{Hechos de la querella}

"Que el día 19 de enero del presente año 2019, en el mirador Prat de Recreo, frente al edificio número 125, de la calle Arturo Prat, ciudad de Viña del Mar, aproximadamente entre las 11:00 y 16:00 horas, don C. A. M. V., ya individualizado, dejó encerrada en el vehículo marca "Subaru", color negro, a una perra conocida como "Castaña", aparentemente de raza Golden Retriever. Lo anterior ocasionó el maltrato, daño y posterior muerte del animal, ello debido a que la perra se encontraba dentro de una caja de transporte dentro del vehículo, el cual, mantenía sus vidrios cerrados, en un horario en que se registran las temperaturas más altas del día. Cabe destacar que el vehículo se encontraba a pleno sol en el mirador ya señalado, lo cual generó un aumento de temperatura, que produciría, a lo menos, un detrimento en la salud, tanto física como psíquica, de un animal que se encontrara en dichas condiciones.

Según testigos y medios de prensa, se intentó contactar al responsable del animal, quien se encontraba en el edificio de en frente. Sin embargo, este sujeto bajó del señalado edificio largo rato después, cuando la canina ya se encontraba muerta.

Ante dicha situación, habiéndose percatado los testigos del riesgo vital que sufría la canina encerrada dentro del vehículo y, a su vez, en la caja de transporte canino, ellos procedieron a quebrar los vidrios del vehículo para rescatar a "Castaña" del interior. Una vez realizada dicha acción, los presentes intentaron reanimar a la canina, quién aún se encontraba con vida al momento de ser sacada del vehículo. Se le proporcionó agua e inclusive respiración boca a boca, siendo todos aquellos esfuerzos inútiles, pereciendo la perrita pocos minutos después de ser sacada del automóvil.

Posterior a lo acontecido, los testigos llaman a Carabineros, quienes llegan aproximadamente treinta

\footnotetext{
${ }^{14}$ EMOL (2019): Perro muere tras permanecer varias horas encerrado en un automóvil en Viña del Mar. En línea: https://www.emol.com/noticias/Nacional/2019/01/21/935069/Perro-murio-tras-pasar-varias-horas-encerrado-en-un-automovil-enVina-del-Mar.html [último acceso el 16 de enero de 2021].

${ }^{15}$ Mestizos Magazine (2019): Habla cuidador que rompió ventana de un auto para sacar a perrita. En línea: https://mestizos.cl/noticias/habla-cuidador-que-rompio-ventana-de-un-auto-para-sacar-a-perrita/ [último acceso el 16 de enero de 2021].
} 
minutos después y toman declaración de los hechos a los presentes. Aproximadamente quince minutos después de la llegada del personal policial, C. A. M. V. baja del edificio y se le toma declaración."

\title{
2.2.2 Resolución del caso
}

El día 2 de diciembre de 2019, se realizaría audiencia de suspensión condicional del procedimiento, con la presencia de la querellante Fundación Derecho y Defensa Animal. Cumpliendo con los requisitos del artículo 237 del Código Procesal Penal ${ }^{16}$, se adoptó la salida alternativa de suspensión condicional del procedimiento, fijándose las siguientes condiciones por un año: Entregar a título de donación la suma de $\$ 1.000 .000$, en 10 cuotas, a una organización de protección animal de la región; fijar domicilio e informar de cambios de éste; e inhabilidad de tenencia de animales por el plazo de un año. Estas condiciones corresponden a las dispuestas en las letras e, $\mathrm{g}$ y h del artículo 238 del Código Procesal Penal ${ }^{17}$. La suspensión condicional del procedimiento, en el sistema de justicia penal chileno, es una forma de término anticipado, evitando la realización de un juicio oral y, de acuerdo con el profesor CERDA "su fundamento político criminal consiste en evitar oportunamente los efectos criminógenos del procedimiento penal y la prisión preventiva respecto de imputados por delitos menores y con bajo o inexistente compromiso delictual previo"18.

Al haberse cumplido el plazo y las condiciones de la suspensión condicional, el Juzgado de Garantía de Viña del Mar sobreseería definitivamente este caso el día 10 de diciembre de 2020, cerrando así este caso sin juicio oral.

\section{Comentario de los casos}

A modo de sumario, al examinar los casos relatados podemos identificar que en ambos la conducta imputada es dejar a perros en el interior de un vehículo en un día en que se registró una alta temperatura, lo cual ocasiona como resultado la muerte de estos animales, hechos subsumibles en el inciso 3 del artículo 291 bis del Código Penal. Por otro lado, ambos casos tienen distintas formas de término, realizándose juicio oral sólo en uno de ellos, mientras que en el otro la forma de término del procedimiento fue una suspensión condicional. Además, si bien en ambos casos hubo rescate, solo en uno se detuvo a quien realizó la conducta de rescate, siendo formalizado y condenado por delito de daños simples del artículo 484 del Código Penal ${ }^{19}$. En atención a lo anterior, a continuación, examinaré las particularidades de cada uno de estos casos.

\subsection{Comentario Caso 1}

\begin{abstract}
${ }^{16}$ Artículo 237 del Código Procesal Penal: “Suspensión condicional del procedimiento. El fiscal, con el acuerdo del imputado, podrá solicitar al juez de garantía la suspensión condicional del procedimiento. El juez podrá requerir del ministerio público los antecedentes que estimare necesarios para resolver. La suspensión condicional del procedimiento podrá decretarse: a) Si la pena que pudiere imponerse al imputado, en el evento de dictarse sentencia condenatoria, no excediere de tres años de privación de libertad; b) Si el imputado no hubiere sido condenado anteriormente por crimen o simple delito, y c) Si el imputado no tuviere vigente una suspensión condicional del procedimiento, al momento de verificarse los hechos materia del nuevo proceso. La presencia del defensor del imputado en la audiencia en que se ventilare la solicitud de suspensión condicional del procedimiento constituirá un requisito de validez de la misma. Si el querellante o la víctima asistieren a la audiencia en que se ventile la solicitud de suspensión condicional del procedimiento, deberán ser oídos por el tribunal. Tratándose de imputados por delitos de homicidio, secuestro, robo con violencia o intimidación en las personas o fuerza en las cosas, sustracción de menores, aborto; por los contemplados en los artículos 361 a 366 bis y 367 del Código Penal; por los delitos señalados en los artículos $8^{\circ}, 9^{\circ}, 10,13,14$ y $14 \mathrm{D}$ de la ley $\mathrm{N}^{\circ} 17.798$; por los delitos o cuasidelitos contemplados en otros cuerpos legales que se cometan empleando alguna de las armas o elementos mencionados en las letras a), b), c), d) y e) del artículo $2^{\circ}$ y en el artículo $3^{\circ}$ de la citada ley $\mathrm{N}^{\circ} 17.798$, y por conducción en estado de ebriedad causando la muerte o lesiones graves o gravísimas, el fiscal deberá someter su decisión de solicitar la suspensión condicional del procedimiento al Fiscal Regional. Al decretar la suspensión condicional del procedimiento, el juez de garantía establecerá las condiciones a las que deberá someterse el imputado, por el plazo que determine, el que no podrá ser inferior a un año ni superior a tres. Durante dicho período no se reanudará el curso de la prescripción de la acción penal. Asimismo, durante el término por el que se prolongare la suspensión condicional del procedimiento se suspenderá el plazo previsto en el artículo 247. La resolución que se pronunciare acerca de la suspensión condicional del procedimiento será apelable por el imputado, por la víctima, por el ministerio público y por el querellante. La suspensión condicional del procedimiento no impedirá de modo alguno el derecho a perseguir por la vía civil las responsabilidades pecuniarias derivadas del mismo hecho."

${ }^{17}$ Artículo 238 del Código Procesal Penal: "Condiciones por cumplir decretada la suspensión condicional del procedimiento. El juez de garantía dispondrá, según correspondiere, que durante el período de suspensión, el imputado esté sujeto al cumplimiento de una o más de las siguientes condiciones: e) Pagar una determinada suma, a título de indemnización de perjuicios, a favor de la víctima o garantizar debidamente su pago. Se podrá autorizar el pago en cuotas o dentro de un determinado plazo, el que en ningún caso podrá exceder el período de suspensión del procedimiento; g) Fijar domicilio e informar al ministerio público de cualquier cambio del mismo, y h) Otra condición que resulte adecuada en consideración con las circunstancias del caso concreto de que se tratare y fuere propuesta, fundadamente, por el Ministerio Público."
\end{abstract}

${ }^{18}$ CERDA SAN MARTIN, R., Manual del sistema de justicia penal tomo I (Santiago, 2013) 285.

${ }^{19}$ Artículo 484 del Código Penal: "Incurren en el delito de daños y están sujetos a las penas de este párrafo, los que en la propiedad ajena causaren alguno que no se halle comprendido en el párrafo anterior." 
En cuanto al caso de Rengo, la sentencia definitiva dictada por el Tribunal de Juico Oral en lo Penal de Rancagua sería absolutoria, pues se rechazaría la imputación de delito de maltrato de animal porque la conducta de los acusados habría sido realizada con culpa con representación y este delito solo se castiga a título doloso. Los elementos para considerar que no hubo dolo fueron principalmente la circunstancia de que los acusados habrían dejado las ventanas abiertas -a lo menos- 10 centímetros, y que el otro can afectado no habría fallecido. De este modo, hubo representación de la conducta y el posible resultado lesivo, pero no habría sido aceptado por los sujetos por cuanto adoptaron medidas para resguardar la integridad de los perros.

La decisión no fue unánime pues el voto en contra estuvo por condenar por delito de maltrato de animal, considerando la concurrencia de dolo eventual, pero solo respecto del resultado de la figura base del artículo 291 bis del Código Penal, esto es, daño, dolor o sufrimiento, no así respecto de la muerte del animal. El razonamiento detrás de este pronunciamiento descansa en que los sujetos, si bien adoptaron medidas para el resguardo de los perros, fueron indiferentes a que se provocara con su conducta los resultados de daño, dolor o sufrimiento, cuestión que no se puede sostener respecto de la muerte de los canes. Así, los sujetos bajan de sus vehículos dejando a los canes encerrados, siendo indiferentes al resultado de la figura base del artículo 291 bis del Código Penal.

Respecto de la discusión de la sentencia, cobra importancia el debate acerca de la distinción entre dolo eventual y culpa con representación. Sabido es por todos que el dolo eventual es aquella clase de dolo en que el sujeto se representa la realización de un hecho típico como consecuencia posible de su conducta y, a pesar de aquello, acepta la eventualidad de que se produzca ${ }^{20}$. Dejando de lado la polémica entre las teorías de la voluntad y de la representación, CURY explica que, si bien el criterio predominante en la actualidad pone acento en la representación del sujeto, también requiere que éste se haya "conformado" con la producción del resultado típico, es decir, que haya decidido por el injusto y lo incorpore en su plan ${ }^{21}$. Así, en palabras del mismo autor: "la presencia de dolo eventual debe afirmarse a partir de la representación de una elevada posibilidad de que el hecho típico se realice y de una decisión de proseguir adelante que es independiente de si el sujeto aprueba ese resultado, bastando con que se "resigne" o se "conforme" con que ocurra" 22 .

De esta forma y siguiendo el criterio explicado por CURY, opino que el razonamiento seguido por el voto minoritario fue el adecuado y que, de acuerdo con la dinámica de los hechos, los acusados de este caso habrían obrado con dolo eventual pues se "resignaron" a que las cosas sucedieran al menos en el sentido de causar daño, dolor o sufrimiento a los perros, pues era previsible que las altas temperaturas de ese día causaran malestares a estos canes al encontrarse encerrados. Por lo anterior, creo que la decisión absolutoria fue inadecuada y lo correcto habría sido una condena por la comisión de la figura base de maltrato de animal del inciso primero artículo 291 bis del Código Penal.

\subsection{Comentario Caso 2}

Por otro lado, respecto del caso "Castaña", como su forma de término fue la salida alternativa de suspensión condicional del procedimiento y sus condiciones fueron cumplidas en su totalidad, no tenemos un pronunciamiento del tribunal analizando el fondo de los hechos, a diferencia del Caso 1. No obstante, es otro el elemento que llama la atención de este caso: el rescate realizado por terceros.

Tal como se comentó en el punto 2.2, en este caso un testigo de los hechos, cuidador de vehículos del sector donde estaba estacionado el automóvil en el cual se encontraba Castaña, intentó rescatar al can rompiendo con una piedra uno de los vidrios del vehículo, siendo posteriormente detenido por el delito de daños simples. En bruto, el problema que tenemos ante nosotros es el siguiente: $\mathrm{X}$ lesiona el derecho de $\mathrm{Y}$, para salvar a $Z$ o, dicho de otra forma, $X$ rompe el vidrio del automóvil de $Y$, para rescatar a $Z$. Si Z fuera un individuo de la especie humana, el centro de atención habría sido que $X$ rescató (o intentó rescatar) una vida humana y lo más probable es que éste no habría sufrido una detención por dañar la propiedad ajena, pero como en este caso $\mathrm{Z}$ era un animal no humano (un can), el foco estuvo en la lesión a la propiedad ajena y, finalmente, $X$ fue detenido por Carabineros, por más que haya intentado salvaguardar los intereses de un individuo dotado de sintiencia.

Expuesto de esta forma, en este caso el problema jurídico que se nos plantea es sobre la justificación de las acciones destinadas a rescatar a un animal no humano encerrado en un "hot car", cuestión que nos lleva al campo de antijuridicidad y las causales de justificación. Tratándose el caso anterior de una colisión de intereses (propiedad privada frente a integridad y vida animal), lo lógico sería contemplar, eventualmente, la

\footnotetext{
${ }^{20}$ CURY URZUA, E., Derecho penal parte general tomo I (Santiago, 2020) 435.

${ }^{21}$ Ibid., p. 441.

${ }^{22}$ Ibid., p. 442.
} 
concurrencia de la causal de justificación de estado de necesidad, del artículo $10 \mathrm{~N}^{\circ} 7$ del Código Penal ${ }^{23}$. El estado de necesidad justificante tiene su fundamento en el principio de interés preponderante ${ }^{24}$, pues busca preservar un derecho en casos de lesión de intereses ajenos para salvaguardar uno propio o también ajeno (en cuyo último caso se habla de "auxilio necesario"), que tiene como elementos: (1) una situación de necesidad, que en palabras de CURY es "la existencia de una situación en la que la salvación de un bien jurídico no es posible sino mediante la realización de una acción típica que causa un mal menor" 25 y (2) un sacrificio necesario, entendido como la causación de un mal sobre bien jurídico ajeno de menor para salvaguardar otro más importante y que este sea el único medio practicable y menos perjudicial para evitar el mal que amenaza, pues esta causal de justificación es subsidiaria ${ }^{26}$.

Si volvemos al problema anterior, que $\mathrm{X}$ rompa el vidrio del automóvil de $\mathrm{Y}$, para rescatar al animal no humano $Z$, puede perfectamente ser amparado bajo el estado de necesidad justificante pues en el caso concreto existía una situación de necesidad ( $Z$ está encerrada en condiciones que su vida corre peligro) y un sacrificio necesario (el único medio idóneo y menos perjudicial para salvarla es afectar la propiedad y romper el vidrio, al mismo tiempo que la vida de un animal no humano claramente tiene mayor valor que un vidrio, que perfectamente puede ser reparado). Lamentablemente, este razonamiento no estuvo presente en los funcionarios que realizaron el operativo policial ni menos en el fiscal de turno, pues se dio la orden de detener en flagrancia al cuidador de vehículos que rompió el vidrio, sin importarles el móvil de sus actos. Con posterioridad, se conocería que este sujeto habría sido condenado a pagar una multa, por delito de daño ${ }^{27}$.

\section{Una mirada al exterior: "hot cars" en EE. UU}

Del análisis de los casos anteriores, podemos concluir que a los "hot cars" le surgen dos problemas jurídicos en nuestro medio: la exigencia de dolo en el delito de maltrato de animal genera que estos casos, al existir dificultades para acreditar dolo eventual, sean considerados como delitos culposos y terminen siendo atípicos, sin generar responsabilidad penal; luego, la posibilidad de rescatar al animal no humano a través de conductas que para ser realizadas requieran afectar propiedad, daría lugar a casos hipotéticos de delito de daños, atribuyéndole responsabilidad penal y civil a quien pretendió rescatar al animal, sin perjuicio de la discutirse la concurrencia de un estado de necesidad. Por otro lado, como adelantamos en el punto 1, los "hot cars" como hipótesis de afectación de la integridad y vida animal, no están regulados en Chile ni como modalidad concreta del delito de maltrato de animal, ni dentro de las leyes sobre protección animal y sobre tenencia responsable.

No obstante, en los Estados Unidos los "hot cars" son contemplados en al menos 31 estados, los cuales cuentan con leyes que prohíben dejar animales encerrados en vehículos en condiciones peligrosas y también normas que otorgan una eximente de responsabilidad a personas que rescatan a un animal en estas circunstancias $^{28}$. Organizaciones como la $\mathrm{ALDF}^{29}$ y la Humane Society of the United States ${ }^{30}$ han trabajado sobre esta temática tanto para sensibilizar sobre los peligros de los "hot cars" en animales no humanos, como también promoviendo cambios legislativos que aborden este tema.

En atención a lo anterior, la ALDF ha estudiado las legislaciones estatales que abordan los "hot cars" y ha encontrado tres tipos de leyes: legislaciones que solo prohíben dejar animales no humanos desamparados en situaciones de riesgo para su salud y vida, legislaciones que además de prohibir permiten que determinados funcionarios puedan rescatar a los animales no humanos realizando acciones que afecten la propiedad y, por último, las "Good Samaritan laws" o leyes del buen samaritano, que eximen responsabilidad a civiles que

\footnotetext{
${ }^{23}$ Artículo 10 del Código Penal: "Están exentos de responsabilidad criminal:

7. ${ }^{\circ}$ El que para evitar un mal ejecuta un hecho, que produzca daño en la propiedad ajena, siempre que concurran las circunstancias siguientes:

Primera. - Realidad o peligro inminente del mal que se trata de evitar.

Segunda. - Que sea mayor que el causado para evitarlo.

Tercera. - Que no haya otro medio practicable y menos perjudicial para impedirlo."

${ }^{24}$ CURY URZUA, E., op. cit., p. 527.

${ }^{25}$ Ibid., p. 550.

${ }^{26}$ Ibid., p. 556.

${ }^{27}$ Mestizos Magazine (2019): Habla cuidador que rompió ventana de un auto para sacar a perrita. En línea: https://mestizos.cl/noticias/habla-cuidador-que-rompio-ventana-de-un-auto-para-sacar-a-perrita/ [último acceso el 16 de enero de 2021].

${ }^{28}$ WISCH, R. F., Table of State Laws that Protect Animals Left in Parked Vehicles, Animal Legal \& Historical Center (2020). En línea: https://www.animallaw.info/topic/table-state-laws-protect-animals-left-parked-vehicles [último acceso el 16 de enero de 2021].

${ }^{29}$ Animal Legal Defense Fund (2019): Acts against leaving dogs locked in hot cars. En línea: https://aldf.org/project/dogs-in-hot-cars/ [último acceso el 16 de enero de 2021].

${ }^{30}$ The Humane Society of the Unites States (s/f): What to do if you see a pet in a parked car. En línea: https://www.humanesociety.org/resources/what-do-if-you-see-pet-parked-car?credit=a6545 [último acceso el 16 de enero de 2021]. 
realicen acciones para rescatar a los animales ${ }^{31}$.

\subsection{Legislaciones que prohíben dejar animales en situaciones de riesgo}

Son normas que prohíben conductas de confinar animales no humanos en vehículos motorizados, generalmente estacionados, en circunstancias que pongan en riesgo la salud, integridad y vida de estos, ya sea por temperaturas extremas, sin ventilación e incluso sin agua ni alimento. Estas normas generalmente son complementarias a los delitos de maltrato animal que poseen estas legislaturas, por lo que no exigen que se produzca algún daño al animal para establecer responsabilidad, la cual puede ser civil o penal dependiendo del estado.

Al menos 17 estados poseerían este tipo de normas, con diferencias entre ellas como establecer excepciones para casos de animales de ganado en California ${ }^{32}$ y Delaware ${ }^{33}$ o derechamente limitar el animal no humano objeto de protección a aquellos de compañía, como en los estados de Dakota del Norte ${ }^{34}$, Dakota del Sur ${ }^{35}$, Maryland ${ }^{36}$, Minnesota ${ }^{37}$, Nevada ${ }^{38}$ y Nuevo Hampshire ${ }^{39}$.

A modo de ejemplo, el Cal. Penal Code $\S 597.7$, de California, dispone:

“597.7. § (a) A person shall not leave or confine an animal in any unattended motor vehicle under conditions that endanger the health or well-being of an animal due to heat, cold, lack of adequate ventilation, or lack of food or water, or other circumstances that could reasonably be expected to cause suffering, disability, or death to the animal."

(...)

(c) Unless the animal suffers great bodily injury, a first conviction for violation of this section is punishable by a fine not exceeding one hundred dollars $(\$ 100)$ per animal. If the animal suffers great bodily injury, a violation of this section is punishable by a fine not exceeding five hundred dollars (\$500), imprisonment in a county jail not exceeding six months, or by both a fine and imprisonment. Any subsequent violation of this section, regardless of injury to the animal, is also punishable by a fine not exceeding five hundred dollars $(\$ 500)$, imprisonment in a county jail not exceeding six months, or by both a fine and imprisonment.

(...)

(f) Nothing in this section shall be deemed to prohibit the transportation of horses, cattle, pigs, sheep, poultry, or other agricultural animals in motor vehicles designed to transport such animals for agricultural purposes."

Por otro lado, el W. Va. Code § 61-8-19, de Virginia Occidental, dispone:

“\$61-8-19. Cruelty to animals; penalties; exclusions.

(a)(1) It is unlawful for any person to intentionally, knowingly or recklessly,

$(\ldots)$

(E) Leave an animal unattended and confined in a motor vehicle when physical injury to or death of the animal is likely to result;

(...)

(2) Any person in violation of subdivision (1) of this subsection is guilty of a misdemeanor and, upon conviction thereof, shall be fined not less than $\$ 300$ nor more than $\$ 2,000$ or confined in jail not more than six months, or both."

\subsection{Legislaciones que permiten rescate por parte de determinados funcionarios}

Por otro lado, en al menos 20 estados existen leyes que permiten que ciertos funcionarios rescaten animales sin enfrentar responsabilidades legales. Entre los funcionarios habilitados para realizar el rescate, en

\footnotetext{
${ }^{31}$ Animal Legal Defense Fund (s/f): An Avoidable Tragedy: Dogs in Hot Cars. En línea: https://aldf.org/project/an-avoidable-tragedydogs-in-hot-cars/ [último acceso el 16 de enero de 2021].

${ }^{32}$ Cal. Penal Code $\S 597.7$.

${ }^{33} 11$ Del.C. $\S 1325$.

${ }^{34} \mathrm{NDCC}, 36-21.2-12$.

${ }^{35}$ SDCL $\S 40-1-36$.

${ }^{36}$ MD Code, Transportation, $\S 21-1004.1$.

${ }^{37}$ M.S.A. $\S 346.57$.

${ }^{38}$ N.R.S. 202.487.

${ }^{39}$ N.H. Rev. Stat. § 644:8-aa.
} 
la mayoría de estos se encuentran funcionarios policiales, de oficinas de control animal, bomberos y personal de servicios de rescate. También algunos estados facultan a médicos veterinarios ${ }^{40}$, guardias de seguridad con licencia ${ }^{41}$ e incluso personal médico servicio de emergencias médicas ${ }^{42}$. En todos estos casos, existe una eximente de responsabilidad por los daños producidos y, por lo general, se exige realizar esfuerzos razonables para localizar al dueño del vehículo, para posterior emplear fuerza proporcionalmente razonable para la finalidad de rescatar al animal, asistirlo y trasladarlo a un refugio u otro lugar, dejando una nota en el vehículo con las indicaciones para recuperar al animal.

Como ejemplo de normas leyes, Maine en el 7 MRSA $§ 4019$ establece:

“\$ 4019. Removal from unattended motor vehicle

1. Removal authorized. A law enforcement officer, humane agent, animal control officer, firefighter as defined in Title 26, section 2101 or security guard licensed under Title 32, chapter 93, referred to in this section as "authorized persons," may take all steps that are reasonably necessary to remove an animal from a motor vehicle if the animal's safety, health or well-being appears to be in immediate danger from heat, cold or lack of adequate ventilation and the conditions could reasonably be expected to cause extreme suffering or death.

2. Notice required. A law enforcement officer, humane agent or animal control officer who removes an animal in accordance with subsection 1 shall, in a secure and conspicuous location on or within the motor vehicle, leave written notice bearing the officer's or agent's name and office and the address of the location where the animal may be claimed. A firefighter, first responder or security guard who removes an animal in accordance with subsection 1 shall, in a secure and conspicuous location on or within the motor vehicle, leave written notice bearing the person's name and the address of the location where the animal may be claimed. The owner may claim the animal only after payment of all charges that have accrued for the maintenance, care, medical treatment and impoundment of the animal.

3. Immunity. An authorized person who removes an animal from a motor vehicle pursuant to subsection 1 is immune from criminal or civil liability that might otherwise result from the removal."

Por otro lado, la N.C.G.S.A. § 14-363.3 de Carolina del Norte señala:

“\$ 14-363.3. Confinement of animals in motor vehicles

(a) In order to protect the health and safety of an animal, any animal control officer, animal cruelty investigator appointed under G.S. 19A-45, law enforcement officer, firefighter, or rescue squad worker, who has probable cause to believe that an animal is confined in a motor vehicle under conditions that are likely to cause suffering, injury, or death to the animal due to heat, cold, lack of adequate ventilation, or under other endangering conditions, may enter the motor vehicle by any reasonable means under the circumstances after making a reasonable effort to locate the owner or other person responsible for the animal.

(b) Nothing in this section shall be construed to apply to the transportation of horses, cattle, sheep, swine, poultry, or other livestock."

\subsection{Legislaciones del "buen samaritano"}

Los estados que poseen una "Good Samaritan law", por lo general otorgan eximente de responsabilidad civil y en algunos casos penales, a cualquier civil que realice acciones para rescatar a un animal generalmente de compañía, en situaciones de peligro a su salud. También los estados de Connecticut ${ }^{43}$, Oregon ${ }^{44}$ y Tennessee ${ }^{45}$ incluyen a los menores de edad dentro de estas situaciones de riesgo.

En este tipo de leyes se establecen una serie de obligaciones o requisitos previos cuando se descubra que un animal está sufriendo un golpe de calor dentro de un vehículo como, por ejemplo, que la primera acción que deba realizarse sea revisar que no exista forma de que el animal pueda salir del lugar sin generar daños a la propiedad y, por otra parte, llamar a determinadas autoridades, como policías, bomberos u otros, para efectuar el rescate. Si las autoridades no responden o tardan demasiado al punto en que se pone en riesgo la vida del animal, la persona podrá decidir rescatarlo y gozar de la eximente de responsabilidad que establezca

\footnotetext{
${ }^{40}$ Este es el caso de Indiana en la I.C. 34-30-30-1-4.

${ }^{41}$ Este es el caso de Maine en el 7 MRSA $§ 4019$

${ }^{42}$ Este es el caso de Virginia en la Va. Code Ann. § 3.2-6504.1.

${ }^{43}$ C. G. S. A. $\S 52-557 \mathrm{u}$.

${ }^{44}$ O. R. S. § 30.813.

${ }^{45}$ T. C. A. $§ 29-34-209$.

130 Derecho Animal. Forum of Animal Law Studies, vol. 12/1
} 
la ley en caso de cometer daños contra la propiedad. La fuerza empleada debe ser aquella proporcionalmente razonable para la finalidad de rescate del animal y por lo general, una vez realizado el rescate, el "buen samaritano" debe permanecer en el lugar con el animal hasta que llegue una autoridad.

Existen 14 estados con este tipo de normas, la mayoría de ellos solo otorgan eximente de responsabilidad civil, pero también otros como California ${ }^{46}$, Colorado ${ }^{47}$, Connecticut, Massachusetts ${ }^{48}$ y Oregon otorgan una eximente de responsabilidad penal, mientras que Indiana establece eximentes para ambos tipos de responsabilidades, pero la civil está limitada solo hasta la mitad del costo de reparación del daño al vehículo motorizado.

A modo de ejemplo, podemos ver que la T. C. A. § 29-34-209, de Tennessee establece:

"§ 29-34-209. Forcible entry of a motor vehicle for purposes of removing a minor or an animal

(a) A person whose conduct conforms to the requirements of subsection (b) shall be immune from civil liability for any damage resulting from the forcible entry of a motor vehicle for the purpose of removing a minor or an animal from the vehicle.

(b) Subsection (a) applies if the person:

(1) Determines the vehicle is locked or there is otherwise no reasonable method for the minor or animal to exit the vehicle;

(2) Has a good faith belief that forcible entry into the vehicle is necessary because the minor or animal is in imminent danger of suffering harm if not immediately removed from the vehicle and, based upon the circumstances known to the person at the time, the belief is a reasonable one;

(3) Has contacted either the local law enforcement agency, the fire department, or a 911 operator prior to forcibly entering the vehicle;

(4) Places a notice on the vehicle's windshield with the person's contact information, the reason the entry was made, the location of the minor or animal, and the fact that the authorities have been notified; (5) Remains with the minor or animal in a safe location, out of the elements but reasonably close to the vehicle, until law enforcement, fire, or another emergency responder arrives; and

(6) Used no more force to enter the vehicle and remove the child or animal from the vehicle than was necessary under the circumstances.

(c) Nothing in this section shall affect the person's civil liability if the person attempts to render aid to the minor or animal in addition to what is authorized by this section."

Por otro lado, el O. R. S. § 30.813, de Oregon, que dispone:

"§ 30.813 Liability of person who enters motor vehicle to remove unattended child or domestic animal; exceptions. (1) As used in this section, "motor vehicle" has the meaning given that term in ORS 801.360 .

(2) A person who enters a motor vehicle, by force or otherwise, to remove a child or domestic animal left unattended in the motor vehicle is not subject to criminal or civil liability if the person:

(a) Before entering the motor vehicle, determines that the motor vehicle is locked or there is no reasonable method for the child or animal to exit the motor vehicle without assistance;

(b) Has a good faith and reasonable belief, based upon the circumstances, that entry into the motor vehicle is necessary because the child or animal is in imminent danger of suffering harm;

(c) Before or as soon as is reasonably practicable after entering the motor vehicle, notifies law enforcement or emergency services;

(d) Uses no more force than is necessary to enter the motor vehicle and remove the child or animal; and

(e) Remains with the child or animal in a safe location, in reasonable proximity to the motor vehicle, until law enforcement, emergency services or the owner or operator of the motor vehicle arrives.

(3) This section does not limit the liability of a person for gross negligence or for reckless, wanton or intentional misconduct.

(4) This section does not limit the liability of a peace officer as defined in ORS 161.015."

Caso especial es el I.C. 34-30-30-1-4, de Indiana, que exige el pago de la mitad de los daños, mientras exime de responsabilidad penal:

\footnotetext{
${ }^{46}$ La responsabilidad penal se encuentra en la Cal. Penal Code $\$ 597.7$, mientras que la responsabilidad civil es mencionada en la Cal. Civ. Code $\S 43.100$.

${ }^{47}$ C.R.S. 13-21-108.4.

${ }^{48}$ M.G.L.A. $140 \S 174 \mathrm{~F}$.
} 
"34-30-30-3 Procedure for removing domestic animal from motor vehicle

Sec. 3. (a) Except as provided in subsection (c), a person who forcibly enters a motor vehicle to remove a domestic animal from the motor vehicle:

(1) is liable to the owner or lessee of the motor vehicle for one-half $(1 / 2)$ of the cost of repairing the damage to the motor vehicle directly caused by the person's forcible entry, unless the owner or lessee of the motor vehicle waives the person's liability under this subdivision; and

(2) is immune from any other criminal or civil liability for other property damage resulting from the person's forcible entry of the motor vehicle;

(...)"

\section{Conclusiones}

1. La creciente importancia de la protección de los animales no humanos nos invita a repensar todas aquellas conductas riesgosas, a través de las cuales los animales no humanos podrían sufrir afectaciones a su integridad y vida. Ya es un hecho notorio de público conocimiento que las sociedades contemporáneas poseen normas de protección animal de diversa naturaleza y, en ellas, es posible identificar principios como el de evitar sufrimientos innecesarios. Frente a una situación que cause sufrimiento a un animal no humano, se nos genera así un problema de índole jurídica y es tarea del derecho otorgarnos respuestas que permitan resolverlo satisfactoriamente.

2. Los golpes de calor en vehículos motorizados, o "hot cars" como se conocen en los EE.UU, son una clase de este tipo de problemas que generan sufrimiento a los animales producto de un actuar negligente o un cumplimiento defectuoso de los deberes de cuidado que poseen los tenedores de animales no humanos. En los casos más graves, estas conductas generan responsabilidad penal, pues los golpes de calor pueden causar graves daños e incluso la muerte a un animal.

3. En Chile, no estamos ajenos a esta realidad, ya que cada verano somos testigos de nuevos casos de muertes o rescates de animales de compañía (principalmente perros) que se encontraban encerrados en vehículos motorizados estacionados, durante días de altas temperaturas. No obstante, son pocos los casos de esta naturaleza que llegan a conocimiento de tribunales de justicia, por lo que en el presente trabajo pudimos analizar dos casos que tuvieron resultados distintos, pero permiten analizar la naturaleza jurídica de esta situación y cuáles son los problemas que se plantean al respecto.

4. Los "hot cars", en cuanto situación que pone en riesgo la integridad, salud y vida de un animal, no tienen una regulación expresa en Chile, por lo que se debe acudir a las reglas generales. Con todo, al momento de abordar estos casos, lo más común es imputar responsabilidad penal por delito de maltrato de animal a quien deja a un animal de compañía en el interior de un vehículo en un día con altas temperaturas. De los dos casos analizados, podemos identificar como problemas de los "hot cars" en nuestro país que: (1) en atención a la exigencia de dolo en el delito de maltrato de animal y a que en muchas ocasiones estos casos ocurren por negligencia, es bastante probable que no se obtengan resultados condenatorios, y (2) el rescate del animal no humano atrapado en el "hot car" generalmente implica cometer daños contra la propiedad, lo cual en nuestra legislación es delito de daños simples, lo que expone a una detención a quien practique el rescate, sin perjuicio de contar con el estado de necesidad como una causal de justificación a probar en un posible juicio.

5. Relacionado a lo anterior y volviendo a los EE.UU, en al menos 31 estados de este país existen normas que regulan los "hot cars". De acuerdo con la ALDF, se distinguen tres clases de leyes que (1) buscan prohibir dejar animales no humanos encerrados en situaciones que corra riesgo la integridad y vida de ellos, (2) permitir a determinados funcionarios a realizar rescate e, incluso, (3) algunas permitirían a civiles realizar los rescates, eximiendo de responsabilidad civil por los daños y en algunos casos también eximiendo la responsabilidad penal de los delitos asociados. En el punto 4 de este trabajo, revisé algunas de estas leyes y sus particulares, por lo que concluyo que, si bien estas normas no son uniformes y presentan diferencias notorias como qué tipo de animal se puede rescatar, en muchos casos estas legislaciones entregan herramientas jurídicas eficaces para proteger la integridad y vida de los animales no humanos al sancionar expresamente la conducta de dejarlos encerrados y facultar el rescate de estos por parte de civiles, sin enfrentar consecuencias legales. De esta forma, las prohibiciones de los estados que contemplan este tipo de leyes, no esperan un resultado concreto en el animal afectado para castigar a los responsables.

6. Dicho todo esto, es pertinente que, de lege ferenda, en Chile adoptemos normas que sancionen expresamente dejar animales encerrados en vehículos motorizados, en situaciones que corra riesgo la integridad y vida de ellos, como también la causación del resultado de muerte de un animal que permanezca en esta situación, por una conducta negligente del sujeto activo. Así como también, se hace necesario la existencia de normas que faciliten el rescate de estos permitiendo tanto a la autoridad como a civiles realizar acciones tendientes a salvar al animal. En cuanto a lo primero, existiendo en el título III de la Ley $\mathrm{N}^{\circ} 20.380$, 
sobre Protección Animal, normas relacionadas al transporte de cualquier clase de animales, sería factible establecer una prohibición a esta conducta, cuya infracción sea de conocimiento de los Juzgados de Policía Local, sin perjuicio de la responsabilidad penal en caso de afectar dolosamente a la integridad o vida del animal. Lo mismo se puede hacer en el título V de la Ley $\mathrm{N}^{\circ} 21.020$, sobre Tenencia Responsable de Mascotas y Animales de Compañía, que establece normas sobre responsabilidad del tenedor de animales de compañía, siendo factible incorporar una prohibición en dicho título. Así, el tenor de este artículo podría ser:

"Se castigará con multa de cinco a treinta unidades tributarias mensuales a quien deje a un animal encerrado, sin vigilancia, en un vehículo motorizado en condiciones que pongan en peligro la salud y bienestar del animal producto del calor, frío, falta de ventilación adecuada, falta de agua o comida, $\mathrm{u}$ otras circunstancias debido a las cuales se podría esperar razonablemente que cause daño, dolor, sufrimiento o muerte al animal. Si producto de estos hechos, se causa la muerte del animal por negligencia, la multa será de treinta a cincuenta unidades tributarias mensuales."

En cuanto a lo segundo, la norma que permita el rescate podría ser incorporada en los mismos cuerpos legales, facultando a las policías, municipalidades y bomberos a proceder al rescate, mientras que a los civiles se le otorgue la misma facultad siempre y cuando se realicen acciones tales como contactar a las autoridades anteriormente indicadas, usar fuerza proporcional para rescatar al animal, otorgarle asistencia al animal y permanecer con él hasta que llegue una autoridad. El articulado podría estar redactado de la siguiente forma:

"Carabineros, las municipalidades y bomberos podrán intervenir en aquellos casos que presencian esta infracción, comprueben que el dueño del vehículo no se encuentra en el lugar y el vehículo está completamente cerrado, para lo cual podrán usar fuerza en el vehículo para entrar en él y retirar al animal.

Cualquier persona, una vez comprobado que el dueño del vehículo no se encuentra en el lugar y el vehículo está completamente cerrado, podrá retirar al animal del vehículo, utilizando fuerza proporcional para esto, siempre y cuando haya alertado previamente el hecho ante la autoridad competente más inmediata. En este caso, la persona que realiza el rescate deberá permanecer junto al animal hasta que llegue la autoridad."

7. Una propuesta de este tipo sería respetuosa con principios como el de subsidiariedad y fragmentariedad del derecho penal, los cuales entienden al derecho penal como un recurso de ultima ratio, que reacciona ante los atentados más graves a aquellos bienes jurídicos dignos de protección penal ${ }^{49}$. De esta forma, la sanción penal en el caso de los "hot cars", solo aparecerá cuando efectivamente haya una lesión de carácter dolosa al bien jurídico integridad y vida del animal no humano afectado por el golpe de calor, estableciéndose que la conducta de dejar animales encerrados en vehículos motorizados en situaciones de peligro para la integridad y vida de ellos solo como una infracción o contravención de conocimiento de los Juzgados de Policía Local, lo mismo para los casos en que se cause la muerte por negligencia. Por otro lado, si bien puede ser cierto que la exención de responsabilidad aquí propuesta cumple con los requisitos del estado de necesidad, no es menos cierto que establecer una norma de este tipo permitiría que actores institucionales como las policías y fiscalías eviten la detención en flagrancia por delitos de daño a quienes practiquen los rescates de animales encerrados.

8. Como reflexión final, como sociedad tenemos que empezar a tomarnos en serio nuestra relación con los animales no humanos y otorgarles el respeto que ellos se merecen en cuanto seres sintientes. Esto implica tanto transitar a una sociedad libre de explotación animal, como también hacernos cargo responsablemente del cuidado de aquellos animales considerados de compañía que necesariamente conviven con nosotros. Así mismo, el derecho debe permitir el comportamiento solidario hacia los animales no humanos en circunstancias en que estos se encuentran en riesgo, pues, volviendo a traer las palabras del juez que pronunció el voto disidente del caso 1: “¿hubieran dejado a un niño encerrado en esas circunstancias? Lo más probable es que no, precisamente por el riesgo que había, pero como eran animales sí lo hicieron, contraviniendo lo que el legislador, recogiendo un clamor social, quiso tutelar". Esto también lo podemos extender a los rescates, pues en el caso 2, si en lugar de Castaña se hubiere tratado de una niña (humana) encerrada en un vehículo, lo más probable es que habrían detenido al responsable de dicha situación, en lugar de quien practicó el rescate.

\section{Bibliografía}

${ }^{49}$ CURY URZUA, E., op. cit., pp. 85-87. 
- BINFA ÁlvareZ, J., Delito de maltrato animal en el Anteproyecto de Nuevo Código Penal de Chile de 2018, dA. Derecho Animal (Forum of Animal Law Studies) 11/3 (2020).

- CERDA SAN MARTIN, R., Manual del sistema de justicia penal tomo I (Santiago, 2013).

- CURY URZUA, E., Derecho penal parte general tomo I (Santiago, 2020).

- GUZMÁN DÁLBORA, J., El delito de maltrato de animales, en Estudios Penales (Santiago, 2009) 241-295.

- MAÑAliCH, J. P., Animalidad y Subjetividad. Los animales (no humanos) como sujetos-dederecho, en Revista de Derecho (Valdivia). Volumen 31, N 2 (2018) 321-337.

- WISCH, R. F., Table of State Laws that Protect Animals Left in Parked Vehicles, Animal Legal \& Historical Center, (2020). En línea: https://www.animallaw.info/topic/table-state-laws-protectanimals-left-parked-vehicles

\section{Legislación}

Chile

- Código Penal.

- Código Procesal Penal.

- Ley $\mathrm{N}^{\circ} 20.380$.

- Ley $\mathrm{N}^{\circ} 21.020$.

\section{Estados Unidos}

- California Code, Civil Code (Cal. Civ. Code).

- California Code, Penal Code Penal (Cal. Penal Code).

- Colorado Revised Statutes (C.R.S.).

- Connecticut General Statutes Annotated (C. G. S. A.).

- Delaware Code (Del.C.).

- Indiana Code (I.C.).

- Maine Revised Statutes Annotated (MRSA).

- Code of Maryland (MD Code).

- Massachusetts General Laws Annotated (M.G.L.A.).

- Minnesota Statutes Annotated (M.S.A.).

- Nevada Revised Statutes (N.R.S.).

- Revised Statutes Annotated of the State of New Hampshire (N.H. Rev. Stat.).

- North Carolina General Statutes Annotated (N.C.G.S.A.).

- North Dakota Century Code (NDCC).

- Oregon Revised Statutes (O. R. S.).

- South Dakota Codified Laws (SDCL).

- Tennessee Code Annotated (T. C. A.).

- Annotated Code of Virginia (Va. Code Ann.).

- Annotated Code of West Virginia (W. Va. Code).

\section{Jurisprudencia}

- Expediente RIT 347-2019, ante el Juzgado de Garantía de Rengo.

- Expediente RIT 341-2019, Tribunal de Juicio Oral en lo Penal de Rancagua.

- Expediente RIT 1063-2019, ante el Juzgado de Garantía de Viña del Mar.

\section{Otras fuentes}

Sitios web

- Animal Legal Defense Fund (2019): Acts against leaving dogs locked in hot cars. En línea: https://aldf.org/project/dogs-in-hot-cars/

- Animal Legal Defense Fund (s/f): An Avoidable Tragedy: Dogs in Hot Cars. En línea: https://aldf.org/project/an-avoidable-tragedy-dogs-in-hot-cars/

- BioBio Chile (2019): "Oye, es mi auto. No lo rompan por favor": dejó encerrado a su perro con $30^{\circ}$ 
C y fue detenida. En línea: https://www.biobiochile.cl/noticias/nacional/region-de-laaraucania/2019/02/01/oye-es-mi-auto-no-lo-rompan-por-favor-dejo-encerrado-a-su-perro-con-30ode-calor-y-fue-detenida.shtml

- Colegio Médico Veterinario (s/f): Cómo Evitar la Insolación en Perros. En línea: https://www.colegioveterinario.cl/noticias/ver.php?id=194

- Cooperativa (2020): Rescatan a cachorro que estaba encerrado en un auto expuesto al intenso calor. En línea: https://cooperativa.cl/noticias/pais/region-de-valparaiso/rescatan-a-cachorro-que-estabaencerrado-en-un-auto-expuesto-al-intenso/2020-12-28/115610.html

- El Ovallino (2020): Detenido por maltrato animal al dejar a su mascota dentro del auto. En línea: http://www.elovallino.cl/policial/detenido-por-maltrato-animal-dejar-su-mascota-dentro-auto

- El Tipógrafo (2019): Rengo: Denuncian grave caso de maltrato animal contra dos perritas que dejaron encerradas en auto. En línea: https:/eltipografo.cl/2019/01/rengo-denuncian-grave-caso-demaltrato-animal-contra-dos-perritas-que-dejaron-encerradas-en-auto

- EMOL (2019): Perro muere tras permanecer varias horas encerrado en un automóvil en Viña del Mar. En línea: https://www.emol.com/noticias/Nacional/2019/01/21/935069/Perro-murio-traspasar-varias-horas-encerrado-en-un-automovil-en-Vina-del-Mar.html

- La Tercera (2020): Ola de calor: cómo cuidar a tus mascotas frente a las altas temperaturas. En línea: https://www.latercera.com/practico/noticia/ola-de-calor-como-cuidar-a-tus-mascotas-frente-a-lasaltas-temperaturas/XSYJS244FNGYBCOWNONZHSL32E/

- Mestizos Magazine (2019): Habla cuidador que rompió ventana de un auto para sacar a perrita. En línea: https://mestizos.cl/noticias/habla-cuidador-que-rompio-ventana-de-un-auto-para-sacar-aperrita/

- Mestizos Magazine (2019): Mujer deja a su perro encerrado en un auto en Conchalí. En línea: https://mestizos.cl/noticias/mujer-deja-a-su-perro-encerrado-en-un-auto-en-conchali/

- Mestizos Magazine (2019): Perro permaneció encerrado por más de una hora en un auto. En línea: https://mestizos.cl/noticias/perro-permanecio-encerrado-por-mas-de-una-hora-en-un-auto/

- SoyQuillota (2020): Justo a tiempo: rescatan a cachorro encerrado en vehículo a pleno sol en La Ligua. En línea: https://www.soychile.cl/Quillota/Sociedad/2020/12/31/687868/Justo-a-tiemporescatan-a-cachorro-encerrado-en-vehiculo-a-pleno-sol-en-La-Ligua.aspx

- The Humane Society of the Unites States (s/f): What to do if you see a pet in a parked car. En línea: https://www.humanesociety.org/resources/what-do-if-you-see-pet-parked-car?credit=a6545 